\title{
A PREVAlÊNCIA de transtornos MENTAIS NOS TRABALHADORES DA APS NO MUNICIPIO DE CURITIBA/PR
}

\author{
Mental health disorders prevalence in Primary Health \\ Care workers of the city of Curitiba/PR
}

Ellen Dalla Costa ${ }^{1}$, Luiz Claudio Sobrinho do Nascimento ${ }^{2}$

\begin{abstract}
1. Enfermeira Residente em Estratégia Saúde da Família. ORCID: https://orcid.org/0000-0002-25442046.

2. Enfermeiro na Secretaria Municipal de Saúde de Curitiba e Mestre na Universidade Positivo. ORCID: https://orcid.org/000-0003-1951-0222

CONTATO: Ellen Dalla Costa | Endereço: Rua Vicente Nicolau Kopytowski, 60, casa 4 | Xaxim | Curitiba-PR | Telefone: (41) 98860-6237 | E-mail: ellendalla@gmail.com
\end{abstract}

COMO CITAR: Costa ED, Nascimento LCS. A prevalência de transtornos mentais nos trabalhadores da APS no municipio de Curitiba/PR. R. Saúde Públ. 2019 Jul;2(1):80-92.

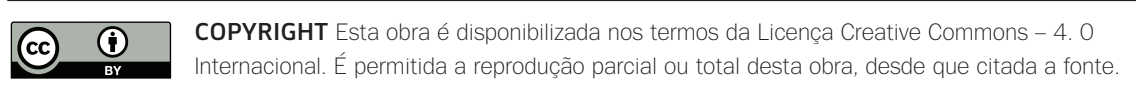

RESUMO Trata-se de uma pesquisa retrospectiva, transversal e descritiva de abordagem quantitativa, cujos objetivos foram pesquisar a prevalência de transtornos de saúde mental nos profissionais de saúde atuantes na APS no município de Curitiba, relacionar essa prevalência com as características epidemiológicas e realizar a comparação das prevalências entre os profissionais das Unidades Básicas de Saúde e os profissionais do modelo de Estratégia Saúde da Família. Para tanto, foram obtidos dados do setor de Saúde Ocupacional da Prefeitura Municipal de Curitiba. As variáveis faixa etária e cargo provaram ser um fator de relevância para o desenvolvimento dos transtornos mentais, e os resultados mostraram uma maior prevalência de afastamento por CID F nos profissionais das UBS do que entre os profissionais da ESF. Faz-se necessário, portanto, o investimento em estratégias de prevenção e tratamento dessas patologias entre os profissionais da APS do município de Curitiba/PR.

PALAVRAS-CHAVE: Saúde do Trabalhador. Saúde Mental. Estratégia Saúde da Família. Atenção Primária à Saúde. 
ABSTRACT This is a retrospective, cross-sectional, and descriptive research of quantitative approach whose objectives were to investigate the prevalence of mental health disorders in health professionals working in Public Primary Health Care in the city of Curitiba, to relate this prevalence with the epidemiological characteristics, and compare it in Basic Health Units and in the units of the Family Health Strategy model. For that, data were obtained from the Occupational Health Department of the City Hall of Curitiba. The variables ge and position proved to be a relevant factor for the development of mental disorders, and the results showed a higher prevalence of mental disorders (ICD-10 F code) within Basic Health Units professionals. Therefore, it is necessary to invest in strategies for the prevention and treatment of these pathologies among the Public Health Care professionals in the city of Curitiba/PR.

KEYWORDS: Occupational Health. Mental health. Family Health Strategy. Primary Health Care.

\section{INTRODUÇÃO}

A satisfação com o trabalho pode ser entendida como um sentimento positivo resultante de fatores favoráveis ao trabalhador, como relações interpessoais bemsucedidas, valorização do trabalho e recursos e ambientes adequados. Além disso, pesquisas realizadas a partir da década de 70 mostraram que outras variáveis como o salário, promoção e supervisão adequadas contribuem para a maior satisfação do trabalhador ${ }^{1.2}$.

Contudo, a subjetividade do processo avaliativo da satisfação do funcionário com seu ambiente de trabalho envolve influências internas e externas a ele, como sua relação familiar, social, lazer, etc., além de suas próprias características e personalidade?2.
Quando os recursos necessários ao trabalho e as demandas do cargo não se encontram ajustadas ao trabalhador, este gera respostas físicas e emocionais que se, por sua vez, estiverem incompatíveis com a condição física e comportamental do indivíduo, surge o estresse $e^{2,3}$.

As respostas do organismo humano frente ao estresse incluem tanto os sintomas físicos quanto os psicológicos. Quanto ao primeiro grupo, os sinais mais comuns incluem sudorese, tensão muscular. hipertensão, taquicardia, náuseas, ranger de dentes, etc. As respostas psicológicas, por sua vez, incluem ansiedade, tensão, sensação de angústia, insônia, preocupação excessiva, dificuldade de relaxar, ira, sensibilidade emotiva e pensamentos exagerados quanto aos fatores estressores ${ }^{4}$. 
O contato direto com o sofrimento de outros seres humanos caracteriza a área da saúde como uma das principais profissões propensas a esse problema ${ }^{2,3}$. Ainda assim, há poucos trabalhos relacionados à saúde dos profissionais de saúde, apesar de ser um cenário de trabalho em que o indivíduo se aproxima constantemente do sofrimento e situações dolorosas ${ }^{5}$.

A percepção de que os profissionais de saúde são muitas vezes vistos como "doadores de saúde" contribui para a falta de medidas e ações voltadas tanto para a prevenção do adoecimento desses indivíduos, quanto para a recuperação de sua saúde, principalmente no âmbito do Sistema Único de Saúde 6 .

Na atenção primária do Brasil, funcionam dois modelos assistenciais: Atenção Básica Tradicional e a Estratégia de Saúde da Família.

A Atenção Básica $(A B)$ é orientada pelos princípios do Sistema Único de Saúde e deve servir como a principal porta de entrada para a rede de saúde. Além disso, possui um papel central na garantia de acesso a uma atenção à saúde de qualidade, conforme o trecho a seguir, encontrado na PNAB?

A atenção básica caracteriza-se por um conjunto de ações de saúde, no âmbito individual e coletivo, que abrange a promoção e a proteção da saúde, a prevenção de agravos, o diagnóstico, o tratamento, a reabilitação, a redução de danos e a manutenção da saúde com o objetivo de desenvolver uma atenção integral que impacte na situação de saúde e autonomia das pessoas e nos determinantes e condicionantes de saúde das coletividades.

As práticas da $A B$ são desenvolvidas sob a forma do trabalho em equipe, sendo que estas assumem a responsabilidade sanitária pela população do seu território definido, levando em consideração todos os seus critérios de risco?

Entre os fundamentos e diretrizes da
Atenção Básica destacam-se a presença de um território adstrito definido, equidade, integralidade e longitudinalidade do cuidado, acolhimento, vinculação, responsabilização e resolutividade. Além disso, deve-se considerar as ações programadas e as de demanda espontânea, tendo em vista a promoção, prevenção, tratamento e reabilitação da saúde. Deve-se, ainda, estimular a participação dos usuários no seu processo de cuidar, estimulando a sua autonomia e a corresponsabilidade do cuidado de sua saúde e da comunidade?.

Como encontra-se na PNAB? a Estratégia Saúde da Família visa a reorganização da atenção básica no País, conforme o trecho a seguir:

\footnotetext{
A Estratégia Saúde da Família [...] é tida como estratégia de expansão, qualificação e consolidação da atenção básica por favorecer uma reorientação do processo de trabalho com maior potencial de aprofundar os princípios, diretrizes e fundamentos da atenção básica, de ampliar a resolutividade e impacto na situação de saúde das pessoas e coletividades [...]
}

Uma característica importante da ESF é a implantação de equipes multiprofissionais nas unidades básicas de saúde. Estas equipes tornamse responsáveis pelo acompanhamento de um número definido de famílias e usuários em um território adstrito?.

As equipes da ESF são formadas por, no mínimo, um médico de família e Comunidade ou médico especialista em Saúde da Família, um enfermeiro generalista ou especialista em Saúde da Família e um auxiliar ou técnico de enfermagem e agentes comunitários de saúde. Pode-se incluir os profissionais de saúde bucal, sendo um cirurgiãodentista generalista ou especialista em Saúde da Família e um auxiliar ou técnico em saúde bucal?.

Por fim, uma das características mais marcantes do modelo da ESF é a possibilidade da criação do vínculo com os usuários do SUS. Esta ligação permite às equipes das unidades de saúde a 
desencadear um processo de transformação social através de estratégias de ações de promoção e prevenção da saúde junto às famílias e comunidade. Portanto, é imprescindível o bem-estar físico, social e mental dos profissionais da ESF para que possam realizar com excelência o seu papel e trazer benefícios tanto para a sua própria vida, quanto para a comunidade $e^{6.8}$.

Neste contexto, a convivência constante com a realidade e as situações de sofrimento dos usuários em conjunto com o vínculo e laços afetivos que se formam no processo do cuidado podem levar o profissional a desencadear o processo de estresse $e^{8.9}$

Sendo assim, identifica-se uma necessidade de estudo acerca dos motivos que levam o trabalhador da Atenção Primária à Saúde (APS) a desenvolver transtornos de saúde mental. A partir disso, pode-se pensar em estratégias especificas para a prevenção e tratamento desses transtornos nesses profissionais.

\section{OBJETIVOS}

Pesquisar a prevalência de transtornos de saúde mental nos profissionais de saúde atuantes na APS no município de Curitiba, relacionar essa prevalência com as características epidemiológicas e comparar entre ESF e UBS.

\section{METODOLOGIA}

Trata-se de uma pesquisa retrospectiva, transversal e descritiva de abordagem quantitativa.

Para sua realização, optou-se por buscar dados presentes no setor de Saúde Ocupacional dos trabalhadores de saúde da SMS de Curitiba.

Esses dados incluem os afastamentos de acordo com a classificação CID-10, especificamente os diagnósticos presentes no subgrupo $F$ (Transtornos mentais e comportamentais). Entre esses dados se encontram o número de profissionais afastados, a quantidade de licenças de saúde homologadas e os dias de afastamento decorrente destas licenças. Além disso, incluem os dados sociodemográficos e epidemiológicos como gênero, faixa etária e cargo. Ressalta-se aqui, que os dados coletados se referem ao período de 2012 a 2017, porém atualizados com o banco de dados de 2018. Por exemplo, os dados referentes à faixa etária foram obtidos com base na idade atual dos servidores. O mesmo vale para as outras variáveis.

Os critérios de inclusão do estudo foram: ser profissional de saúde atuante em uma das 111 unidades de saúde do município ou compor o Núcleo de Atenção de Saúde da Família (NASF). Optou-se por pesquisar os servidores com um mínimo de 10 anos de trabalho pela SMS, e o tempo de serviço apresenta-se como preditor para o adoecimento mental, devido ao maior tempo exposto aos elementos laborais desfavoráveis ${ }^{10}$.

Critérios de exclusão: profissionais de saúde atuantes há menos de 10 anos na prefeitura, atuantes nos CAPS, maternidades, UPAS, centros de especialidades médicas, centros de especialidades odontológicas, Centro de Orientações e Aconselhamentos (COA), ambulatórios, Centro de Convivência, Hospitais municipais e Laboratório Municipal.

Após a coleta dos dados concedidos pelo setor de Saúde Ocupacional, os mesmos foram organizados e enviados para uma análise estatística a fim de comprovar resultados estatisticamente significativos para a pesquisa.

Os dados recebidos estavam divididos por grupos, sendo eles: o total de servidores afastados, total de licenças homologadas e o total de dias de afastamento em decorrência dessas licenças. Todos esses grupos foram subdivididos entre tipo de unidade, cargo, faixa etária e gênero.

Deve-se considerar que cada servidor pode estar inserido em mais de uma licença, assim como 
pode ter sido afastado por mais de um diagnóstico, possuindo então mais de um CID F na causa de afastamento.

Na primeira etapa da análise de dados, foi realizada a comparação geral dos dados da variável "tipo de unidade", ou seja, entre ESF e UBS. Para tanto, utilizou-se o teste não paramétrico de Kruskal-Wallis, o qual evidenciou a diferença estatisticamente significativa com 95,25\% de certeza entre a proporção de servidores afastados.

O intervalo de confiança indicado pelo teste de Mann-Whitney confirmou, com alpha 0,05, os resultados encontrados pelo teste de KruskallWallis.

Em relação à análise por gênero, a fim de utilizar todo o conjunto de dados e superar a grande variância proporcionada pela interferência do fator "tipo de unidade" nos dados, foram testadas as transformações raiz quadrada, inversa e log. A única a regularizar a variância de maneira a garantir as suposições de normalidade e homogeneidade das variâncias em todas as comparações foi a transformação log, que então foi mantida para a análise dos dados. Foi utilizado em seguida intervalo de confiança 95\% do teste $t$ para desvios de padrões iguais.

Para as análises por cargo e faixa etária foram utilizados os testes de Kruskal-Wallis para análise dos conjuntos completos e, em seguida, os testes de Unweighted pair-group average, que compara a distância média entre todos os membros de todos os grupos, montando a árvore dos mais próximos aos mais distantes e o método de Ward, que consiste na combinação dos grupos de forma a ter o menor aumento possivel da variância.

A comparação das duas metodologias permitiu uma melhor observação do comportamento dos grupos, o que possibilitou a formação das hipóteses iniciais testadas por meio dos intervalos de confiança do teste de Mann-Whitney. A confirmação dessas hipóteses dá-se pela não diferença entre membros de um mesmo grupo e subsequente confirmação da diferença entre agrupamentos.
Especificamente para a análise dos cargos, devido à aparente existência de diferentes títulos a uma mesma função, quando a estrutura dos dados se mantinha a mesma entre dois cargos e a ausência de média anual era simultânea à presença desta no outro cargo de título semelhante, estes foram combinados. Foi o caso dos seguintes cargos: técnico em saúde bucal e técnico em saúde bucal em saúde pública, auxiliar de saúde bucal e auxiliar de saúde bucal em saúde pública e auxiliar de enfermagem com técnico de enfermagem em saúde pública.

Ressalta-se, aqui, que foram excluídos da análise nas UBS os cargos "Assistente de desenvolvimento social", que tinha médias anuais somente para os anos de 2012 e 2014 e um só funcionário; e "Fisioterapeuta" com média anual somente para o ano de 2014 e três funcionários no quadro atual.

Para a análise das ESF foram excluídos os cargos "Agente administrativo" e "Auxiliar administrativo operacional", devido à existência de médias anuais somente dos anos de 2012 e 2013 e à ausência de funcionários nestas funções no quadro atual de funcionários.

Quanto à análise de idade, foi excluída a faixa de 26 a 30 anos, devido à presença de médias anuais somente às UBS para os anos de 2013 e 2014 e 3 funcionários no quadro atual.

Este trabalho foi aprovado pelo Comitê de Ética da Prefeitura Municipal de Curitiba sob o parecer número 2.609.869, e de acordo com a Resolução n 466 de 12 de setembro de 2012 do Conselho Nacional de Saúde.

\section{RESULTADOS}

A pesquisa foi realizada considerando os profissionais das 111 Unidades Municipais de Saúde do município e foram consideradas as quantidades de servidores conforme a tabela 1. 
Tabela 1 Total de servidores atuantes nas Unidades de Saúde de Curitiba há mais de 10 anos, segundo o quadro de funcionários de 2018.

\begin{tabular}{ccc}
\hline Tipo de Unidade & $\mathbf{N}^{\circ}$ de servidores & $\%$ \\
\hline ESF & 1503 & 53,89 \\
\hline UBS & 1286 & 46,11 \\
\hline Total & 2789 & 100 \\
\hline
\end{tabular}

Fonte: Prefeitura Municipal de Curitiba, 2018

A tabela 2 mostra os dados utilizados para a comparação geral da proporção de afastamentos por CID F entre as unidades do tipo ESF e UBS.

Tabela 2 Servidores afastados, licenças homologadas e dias de afastamento por CID F entre os servidores atuantes nas Unidades de Saúde de Curitiba há mais de 10 anos.

\begin{tabular}{lllllll}
\hline & $\mathbf{2 0 1 2}$ & $\mathbf{2 0 1 3}$ & $\mathbf{2 0 1 4}$ & $\mathbf{2 0 1 5}$ & $\mathbf{2 0 1 6}$ & $\mathbf{2 0 1 7}$ \\
\hline $\begin{array}{l}\text { Total de Servidores Afastados } \\
\text { ESF }\end{array}$ & 246 & 272 & 236 & 228 & 162 & 183 \\
\hline UBS & 142 & 150 & 135 & 136 & 121 & 127 \\
\hline Total & $\mathbf{3 8 3}$ & $\mathbf{4 1 5}$ & $\mathbf{3 6 8}$ & $\mathbf{3 6 4}$ & $\mathbf{2 7 9}$ & $\mathbf{3 1 0}$ \\
\hline
\end{tabular}

Total de Licenças Homologadas

\begin{tabular}{|c|c|c|c|c|c|c|}
\hline ESF & 513 & 603 & 586 & 515 & 312 & 384 \\
\hline UBS & 356 & 401 & 351 & 277 & 249 & 287 \\
\hline
\end{tabular}

Total de Dias de Afastamento

\begin{tabular}{lllllllll} 
ESF & 4.647 & 5.693 & 5.448 & 4.598 & 2.582 & 3.388 \\
\hline UBS & & 4.505 & 4.476 & 3.838 & 2.844 & 2.738 & 3.749 \\
\hline Total & $\mathbf{9 . 1 5 2}$ & $\mathbf{1 0 . 1 6 9}$ & $\mathbf{9 . 2 8 6}$ & $\mathbf{7 . 4 4 2}$ & $\mathbf{5 . 3 2 0}$ & $\mathbf{7 . 1 3 7}$ \\
\hline
\end{tabular}


A primeira etapa da análise de dados comparou a proporção de servidores afastados entre ESF e UBS. Para tanto, utilizou-se o teste não paramétrico de Kruskal-Wallis, o qual evidenciou que a mediana da proporção de servidores afastados no ano foi de 15\% para as ESF e 35,4\% para as UBS, diferença estatisticamente significativa com 95,25\% de certeza.

Por sua vez, a análise quanto à quantidade de licenças homologadas e dos dias de afastamento divididos por servidor não obtiveram diferenças estatisticamente significativas entre ESF e UBS, conforme o teste de Kruskal-Wallis.

Para a análise quanto aos dias de afastamento por licença homologada, o teste de Kruskall-Wallis rejeitou a hipótese de igualdade das medianas, pois o percentual foi menor do que 5\%. 0 intervalo de confiança indicado pelo teste de Mann-Whitney confirmou, com alpha 0,05, o encontrado pelo teste de Kruskall-Wallis, ou seja, o tempo de afastamento por licença homologada também obteve resultado estatisticamente significativo, sendo que cada licença durou, em média, 7 dias (UBS) contra 5,5 dias (ESF). Anualmente, esse número passa para 12 e 9 dias, respectivamente.

Quanto aos dias de afastamento por servidor afastado, não foram obtidas diferenças estatisticamente significativas.

A relevância do gênero, após a análise, não pôde ser provada como significativo fator de diferenciação para este estudo.

Para as análises por cargo, devido ao diferente conjunto de dados de um mesmo cargo em diferentes unidades, optou-se por realizar os testes separadamente para ESF e UBS.

Tabela 3 Total de servidores atuantes há mais de 10 anos nas Unidades de Saúde de Curitiba, divididos por cargo e tipo de unidade.

\begin{tabular}{|c|c|c|}
\hline CARGO & ESF & UBS \\
\hline Agente administrativo & 0 & 98 \\
\hline Assistente de desenvolvimento social & 0 & 1 \\
\hline Assistente social & 1 & 0 \\
\hline Atendente de secretaria & 0 & 1 \\
\hline Auxiliar administrativo operacional & 0 & 54 \\
\hline Auxiliar de saúde bucal em saúde pública & 214 & 147 \\
\hline Cirurgião dentista & 161 & 109 \\
\hline Educador social & 0 & 2 \\
\hline Enfermeiro & 171 & 153 \\
\hline Farmacêutico-bioquímico & 23 & 5 \\
\hline Fisioterapeuta & 29 & 3 \\
\hline Fonoaudiólogo & 4 & 0 \\
\hline Médico & 228 & 207 \\
\hline Nutricionista & 9 & 1 \\
\hline Orientador em esporte e lazer & 25 & 3 \\
\hline Psicólogo & 30 & 5 \\
\hline Técnico de enfermagem em saúde pública & 532 & 450 \\
\hline Técnico de saúde bucal em saúde pública & 76 & 46 \\
\hline
\end{tabular}


Em relação às prevalências, no tipo de unidade ESF, o cargo de Enfermeiro apresentou o maior índice de prevalência de afastamentos, com uma diferença de $2,3 \%$ a $11,1 \%$ maior em relação aos outros cargos.

Para as UBS, apesar de observada uma prevalência maior de afastamentos no cargo técnico de enfermagem, foi possível obter um resultado estatisticamente significativo apenas em relação aos técnicos de saúde bucal, que em 95\% dos casos apresentou uma diferença de $-2,2 \%$ a -9,8\% da prevalência de afastamentos em relação aos demais cargos.

A análise da quantidade de licenças homologadas por servidor no tipo de unidade ESF foi feita, também, através de uma avaliação entre grupos, que contrasta os conjuntos [farmacêuticobioquímico, fisioterapeuta, técnico de saúde bucal e orientador em esporte e lazer] contra o grupo que contém os demais cargos.

Confirmou-se a diferença significativa entre os dois conjuntos de cargos, de 9 a 22 licenças médicas a menos, por ano, para cada 100 servidores neste grupo composto pelos 4 cargos listados em relação aos demais. O contraste destes cargos individualmente aos cargos fora deste grupo listado apresenta de 14 a 35 afastamentos a menos a cada 100 servidores por ano, solicitados pelos farmacêuticos-bioquímicos; 13 a 33 afastamentos a menos a cada 100 servidores por ano solicitados por fisioterapeutas; 8 a 27 afastamentos a menos a cada 100 servidores por ano solicitados por técnicos de saúde bucal; e 19 a 38 afastamentos a menos a cada 100 servidores por ano solicitados por orientadores em esporte e lazer.

Essa análise no tipo de unidade UBS não obteve diferença significativa entre os cargos.

Em relação aos dias de afastamento por servidor da ESF realizou-se também uma avaliação entre grupos, que contrasta os conjuntos [farmacêutico-bioquímico, fisioterapeuta, técnico de saúde bucal, nutricionista e orientador em esporte e lazer] contra o grupo que contém os demais cargos.
Confirmou-se a diferença significativa entre os dois conjuntos de cargos, de 1,9 a 3,4 dias de afastamento a menos, por funcionário, por ano. O contraste destes cargos individualmente aos cargos fora deste grupo listado apresentou, por ano, de 1,2 a 3,9 dias de afastamento a menos pelos farmacêuticos-bioquímicos; 1,5 a 4 dias de afastamentos a menos por fisioterapeutas e nutricionistas; 1,3 a 3,8 dias de afastamento a menos por técnicos de saúde bucal; e 1,7 a 4,2 dias de afastamento a menos por orientadores em esporte e lazer.

Para a UBS, também não obteve resultados significativos.

Em relação à duração das licenças, nas unidades do tipo ESF observou-se proximidade nos intervalos a partir da comparação às medianas dos cargos. O cargo de nutricionista e de técnico de saúde bucal foram os únicos que apresentaram diferenças estatisticamente significativas nesta análise, apresentando 3,5 a 8,5 dias a menos de duração por licença médica para os nutricionistas e 1,7 a 6,7 dias a menos por licença médica para o cargo de técnico de saúde bucal.

Para as UBS, o cargo de psicólogo apresentou uma diferença de 8,5 a 13,3 dias a menos por licença médica em relação aos demais cargos (com exceção do agente administrativo e auxiliar administrativo operacional); o agente administrativo teve uma diferença de 4,5 a 8,9 dias a menos por licença; e o auxiliar administrativo operacional apresentou uma diferença de 4,2 a 8,6 dias a menos por licença médica.

O cargo de enfermeiro apresentou afastamento com duração acima do comum nas UBS, de 1 a 11,8 dias por licença médica a mais do que os outros cargos, apesar de estar entre os cargos que apresentaram menor duração das licenças.

Quanto ao tempo de afastamento por cada servidor, na ESF observou-se a real formação de dois grupos, sendo um composto pelos cargos técnico de saúde bucal, nutricionista, orientador 
em esporte e lazer e fisioterapeuta, e o outro pelos cargos auxiliar de saúde bucal, cirurgiãodentista, enfermeiro, farmacêutico-bioquímico, técnico de enfermagem e médico, entre os quais houve diferença significativa, de 9 a 18 dias de afastamento por servidor que solicita a licença, ao ano. Deste primeiro grupo ao cargo de psicólogo, a mediana da diferença e o limite inferior foi 8 dias maior, com um intervalo de diferença entre 2,8 e
23,9 dias de afastamento por servidor afastado.

Para as UBS, foi possivel a análise apenas para o cargo de psicólogo, que apresentou um intervalo de -8,7 a -39 dias de afastamento por servidor em comparação aos outros cargos.

Para a análise por faixa etária, aplicou-se a mesma metodologia utilizada para a análise de gênero.

Tabela 4 Total de servidores atuantes há mais de 10 anos nas Unidades de Saúde de Curitiba, divididos por faixa etária e tipo de unidade.

\begin{tabular}{|c|c|c|c|}
\hline FAIXA ETÁRIA & ESF & UBS & TOTAL \\
\hline Entre 26 e 30 anos & 0 & 3 & 3 \\
\hline Entre 31 e 35 anos & 49 & 71 & 120 \\
\hline Entre 36 e 40 anos & 176 & 177 & 353 \\
\hline Entre 41 e 45 anos & 236 & 229 & 465 \\
\hline Entre 46 e 50 anos & 294 & 274 & 568 \\
\hline Entre 51 e 55 anos & 363 & 252 & 615 \\
\hline Entre 56 e 60 anos & 222 & 162 & 384 \\
\hline Entre 61 e 65 anos & 113 & 75 & 188 \\
\hline Entre 66 e 70 anos & 44 & 37 & 81 \\
\hline Acima de 70 anos & 6 & 6 & 12 \\
\hline
\end{tabular}

Fonte: Prefeitura Municipal de Curitiba, 2018

Quanto ao percentual de servidores afastados, nenhuma das faixas apresentou diferença significativa em relação às outras. Portanto, não se pode afirmar diferença significativa de uma faixa a todas as demais no quesito prevalência de afastamento.

Contudo, a faixa etária de 36 a 40 anos apresentou de 5 a 23 licenças médicas a mais a cada 100 funcionários em relação às outras faixas de idade e de 6,2 a 20,8 dias a mais de afastamento por servidor afastado do que os demais funcionários.
Por sua vez a faixa etária de 61 a 65 anos apresentou de 5 a 18 licenças médicas a menos para cada 100 funcionários, enquanto que o grupo compreendendo a faixa etária de 61 a 70 anos apresentou de 5 a 13,8 dias a menos de afastamento por funcionários do que as demais faixas etárias.

Além disso, quando no mesmo grupo, as faixas etárias de 36 a 40 e de 51 a 55 anos apresentaram uma diferença de 1,7 a 3,1 dias de afastamento a mais por servidor, em comparação com as outras faixas de idade. 


\section{DISCUSSÃO}

Proporcionalmente, a quantidade de servidores afastados por CID $F$ se mostrou maior entre os profissionais das UBS (35,4\%) do que entre os profissionais que trabalham na ESF (15\%). Além disso, o tempo de afastamento por licença homologada também obteve resultado estatisticamente significativo, sendo que cada licença teve duração, em média, de 7 dias (UBS) contra 5,5 dias (ESF). Anualmente, esse número passa para 12 e 9 dias, respectivamente.

Por sua vez, não houve diferença estatisticamente significativa em relação à quantidade de licenças homologadas e dos dias de afastamento por cada servidor.

Segundo Silveira et $\mathrm{al}^{10}$, a diferença de atuação preconizada pela Política Nacional de Atenção Básica, a qual pressupõe que nas unidades do modelo ESF exista, além da territorialização, equipe mínima por número de habitantes, e uma presença maior de agentes comunitários de saúde é um fator que pode trazer melhores condições de trabalho aos seus funcionários em comparação com os funcionários das Unidades Básicas de Saúde.

Um estudo realizado em Porto Alegre concluiu que quanto ao local de atuação, mais de 50\% dos profissionais trabalhavam na ESF e o restante nas UBS, o que corrobora com os achados desta pesquisa. No entanto, nessa pesquisa 37\% dos profissionais da ESF foram afastados por algum tipo de adoecimento, contra 15,2\% dos trabalhadores da UBS $^{10}$.

A pesquisa de Lima ${ }^{11}$ mostrou que a satisfação no trabalho pode ser influenciada, principalmente, por três fatores, sendo eles: "gostar do que se faz", a resolubilidade da assistência e o trabalho em equipe. No contexto da ESF, o vínculo entre equipe e usuários mostrou-se como fonte importante de satisfação com o trabalho.

Além disso, a dupla jornada de trabalho pode consistir em um fator determinante na ocorrência de transtornos mentais ${ }^{12,13,14}$. Devido à carga horária semanal menor entre os trabalhadores das UBS contra a carga horária de 40 horas pelos profissionais da ESF, além da diferença salarial, especula-se que os profissionais das UBS possuam dupla jornada de trabalho, além das tarefas domésticas, o que pode contribuir para deixá-los vulneráveis ao surgimento de transtornos psíquicos.

Outras pesquisas concluíram que o vínculo e o trabalho no território, apesar de constituírem fatores essenciais para o trabalho da ESF, deixam os profissionais vulneráveis ao sofrimento psíquico devido ao contato frequente com o sofrimento alheio e outras situações ${ }^{8.11}$. Isso pode se tornar patológico e trazer, como consequência, transtornos como depressão, síndrome do pânico, abuso de álcool e outras substâncias psicoativas, sendo essas últimas estratégias de fuga para o problema em $\mathrm{si}^{2,4,10,12,13}$.

Apesar do processo que leva ao adoecimento mental constituir-se de múltiplos fatores, os associados ao trabalho, com a falta de condições adequadas para a realização das atividades, falta de autonomia para resolver os problemas, a sobrecarga de trabalho, a pressão advinda da gestão, a carência de recursos humanos e o déficit de instrumentos e de área física constituem as principais causas do adoecimento mental ${ }^{11,14-20}$. Para os trabalhadores da ESF, esses últimos constituem o principal fator de insatisfação com o trabalho 23,8,11,13.

Além dos fatores acima, motivos de insatisfação com o trabalho incluem a questão salarial e as dificuldades nas relações tanto para a realização do trabalho em equipe, quanto com os usuários e as famílias ${ }^{11,19}$.

A variável de gênero não obteve fator significativo de relevância para o estudo, corroborando a pesquisa de Braga7. No entanto, estudos afirmam que a variável gênero interfere na resposta dos indivíduos aos problemas no trabalho, sendo a predominância de médias no gênero feminino $2,4,13,14,21,22$.

Quanto às análises por cargos, vale ressaltar que a utilização de amostras de pequeno tamanho 
amostral fez com que fosse possível provar somente a diferença entre grupos de grande diferença. Este efeito, causado pela utilização de médias anuais, pode ter sido o motivo da não possibilidade de provar a diferença entre diversos cargos. O mesmo ocorreu com o estudo de Batista de Albuquerque ${ }^{23}$, que não conseguiu provar diferenças estatisticamente significantes nas ocorrências de transtornos mentais comuns nas diferentes categorias profissionais.

Nas UBS, destacou-se a presença dos psicólogos abaixo da tendência central na duração das licenças médicas, mantendo distribuição e quantidade de licenças entre os funcionários. Além disso, os cargos de agente administrativo e auxiliar administrativo operacional também apresentaram menor duração das licenças.

Os técnicos de saúde bucal apresentaram a menor prevalência de afastamentos nas UBS corroborando estudos, o que pode ser explicado pelo fato de um trabalho menor com a comunidade ${ }^{23}$. Por sua vez, o cargo que apresentou menor prevalência de afastamentos no tipo de unidade ESF foi orientador em esporte e lazer.

Apesar de, nas UBS, o cargo dos enfermeiros não estar entre os que obtiveram diferença estatística relevante em relação aos demais cargos, observou-se uma maior duração das licenças médicas para os mesmos, sendo de 1 a 11,8 dias a mais por licenças em relação aos outros cargos.

O contrário ocorreu no tipo de unidade ESF. em que os enfermeiros apresentaram a maior proporção de afastamentos em relação às outras categorias profissionais, apesar de não apresentar resultado estatístico relevante em relação à duração e quantidade das licenças.

Estudos ${ }^{10,15,24}$ corroboram com o resultado desta pesquisa em relação aos enfermeiros, estando presente no ranking entre as categorias profissionais que apresentam a maior proporção de transtornos mentais.

Os principais fatores para o adoecimento mental dos enfermeiros são aqueles relacionados às condições - ou falta delas - de trabalho, como estrutura física, ausência de materiais, questão salarial, etc ${ }^{11,16}$. Deve-se considerar o fato de caber ao enfermeiro as funções de gerenciamento, organização e controle do processo de trabalho nas unidades, além, é claro, do contato constante com as condições que podem levar ao adoecimento, como, por exemplo, o contato com as áreas de pobreza extrema, principalmente nas periferias, onde se encontram a maior parte das unidades do tipo ESF $2,15,24$.

Por fim, em relação às análises por faixa etária, o intervalo de 36-40 anos obteve um comportamento diferente em relação aos demais, apresentando maior quantidade de licenças médicas, maior tempo de afastamento e maior tempo de afastamento por servidor afastado, o que vai de encontro com a literatura 10,13,15,17,22,24.

A faixa de 61-65 anos também chamou a atenção com uma menor quantidade de licenças médicas e menor tempo de afastamento por servidor afastado, juntamente com a faixa etária de 66 a 70 anos.

Foi também provada a diferença, mesmo que sutil da quantidade superior de licenças homologadas para a faixa de 51-55, faixa que ficou acima da mediana em todos os testes, o que mostra sua leve superioridade à mediana as respostas.

A prevalência dos transtornos mentais na faixa etária mais jovem pode estar atribuída a pouca experiência do trabalhador e do choque com a realidade ao se deparar com o trabalho a ser exercido ${ }^{17.18}$. Vale ressaltar, porém, que a literatura diverge quanto à prevalência destes transtornos. Ora está relacionado com o maior tempo de serviço ${ }^{10}$, ora está relacionado com a falta de experiência dos mais jovens e suas dificuldades de adaptação ${ }^{17,24}$.

\section{CONSIDERAÇÕES FINAIS}

A proporção de servidores afastados por CID 
F se mostrou maior entre os profissionais das UBS, assim como a duração das licenças. As variáveis cargo e faixa etária também se mostraram serem fatores relevantes para o desenvolvimento de transtornos mentais, sendo a faixa etária jovem de até 40 anos a que apresentou a maior proporção de afastamentos por doença CID F.

O cargo de enfermeiro se destacou em relação aos demais, sendo a categoria profissional que apresentou alta prevalência de afastamento e de duração das licenças. Isso demonstra a realidade vivida por esses profissionais e a necessidade de um olhar diferenciado para essa categoria.

Por fim, os resultados dessa pesquisa demonstram a necessidade da formação de estratégias de prevenção e controle destes transtornos dentre os profissionais da Secretaria Municipal da Saúde, mais especificamente no campo da Atenção Primária â Saúde, de forma a diminuir o absenteísmo por transtornos mentais, além de refletir na melhoria e na qualidade da assistência prestada por esses profissionais.

\section{REFERÊNCIAS}

1. Sampaio JR. Qualidade de vida no trabalho: perspectivas e desafios atuais. Rev Psicol Organ e Trab. 2012;12(1):121-36.

2. Suehiro ACB, Santos AAA, Hatamoto, CT: Cardoso MM Vulnerabilidade ao estresse e satisfação no trabalho em profissionais do Programa de Saúde da Família. Bol Psicol [Internet]. 2008 Dez[citado 2019 Maio 8]:58(129):205-18. Disponivel em: http://pepsic.bvsalud.org/scielo.php?pid=S0006$59432008000200008 \&$ script=sci_arttext.

3. Naiara I, Soares L, Cavalcante L, Souza G, Feitosa A, Castro L, et al. Análise do Estresse Ocupacional e da Síndrome de Burnout em Profissionais da Estratégia da Saúde da Família no Município de Maceió/ AL. Rev Semente. 2011;6(6):84-98.

4. Camelo SHH, Angerami ELS. Sintomas de estresse nos trabalhadores atuantes em cinco núcleos de saúde da família. Rev Lat Am Enfermagem. 2004;12(1):14-21.

5. Alves CFO. Entre o cuidar e o sofrer: o cuidado do cuidador via experiência de cuidadores/ profissionais de saúde mental [dissertação]. Recife: Universidade Católica de Pernambuco:2005.

6. Braga CC, Forte FDS, Melo ACBV. Acessibilidade ao serviço de saúde bucal na atenção básica: desvelando o absenteísmo em uma Unidade de Saúde da Família de João Pessoa-PB. R bras ci Saúde.
2011:15(3):309-18.

7. Brasil. Portaria n² 2.036, 21 setembro 2017. Aprova a Política Nacional de Atenção Básica, estabelecendo a revisão de Diretrizes para a organzação da Atenção Básica, no âmbito do Sistema Único de Saúde (SUS). Disponivel: http://bbvsms.saúde.gov.br/bvs/ saúdelegis/gm/2017/prt2436_22_09_2017.html. Acesso em 20 de abril 2018

8. Silva SS, Martins DC, Camilo LSS. Riscos ocupacionais entre a equipe multidisciplinar na Atenção Básica. International Nursing Congress, 2017,Maio 9-12; Barcelona, Espanha.1-3.

9. Camelo SHH, Angerami ELS. Riscos psicossociais no trabalho que podem levar ao estresse: uma análise da literatura. Ciência Cuid e Saúde. 2008;7(2):232-40

10. Silveira S, Amazarray MR. Preditores da Síndrome de Burnout em profissionais da saúde na atenção básica de Porto Alegre / RS. Cad saúde colet. [Internet].2014 [citado 2018 Abr 22]:22(4):386-392. Disponivel: http://dx.doi.org/10.1590/1414$462 \times 201400040012$

11. Lima L, Pires DEP. Forte ECN, Medeiros F. Satisfação e insatisfação de profissionais de saúde da atenção básica. Esc Anna Nery [Internet]. 2014 Jan-Mar [citado 2018 Abr 22]:18(1):17-24. Disponivel em: http://www.redalyc.org/articulo.oa?id=127730129026. 2014

12. Nogueira-Martins LA. Saúde mental dos profissionais de saúde. Rev Bras Med Trab Belo Horiz. 2003 Jul-Set;1(1):56-68.

13. De Jesus $M$ et al. Abordagem multifatorial do absenteísmo por doença em trabalhadores de enfermagem. Rev Saúde Pública. 2012:46(2):259-68

14. Santos VC, Ferreira SMS, Júnior DFM, Sobrinho CLN, et al Trabalho e saúde mental dos profissionais da Estratégia Saúde da Família em um município do Estado da Bahia, Brasil. Rev Bras Saúde ocup São Paulo. 2012:37(126):306-15.

15. Trindade LL, Lautert L, Beck CLC, Amestoy SC, Pires DEP, et al. Stress and burnout syndrome among workers of the Family Health team . Acta paul. enferm. 2010 Set-Out;23(5):684-9.

16. Miquelim JDL, Carvalho CBO, Gir E, Pelá NTR. Estresse nos profissionais de enfermagem que atuam em uma unidade de pacientes portadores de HIV-AIDS. J Bras Doenças Sex Transm 2004:16(3):24-31.

17. Santana G, Carreiro GSP. Ferreira Filha MO, Lazarte R, Silva AO et al. O processo de adoecimento mental do trabalhador da Estratégia Saúde da Família. Rev Eletr Enf [Internet]. 2013 Jan-Mar [citado 2018 Abr 25]:15(1):146-55. Disponivel em :http://dx.doi. org/10.5216/ree.v15i1.14084

18. Medeiros PA, Silva LC, Amarante IM, Cardoso VG, Mensch KM, Naman M, Chequim L, Silva DA, De Amarante IM, Schimith MD. Condições de saúde entre profissionais da atenção básica em saúde do município de Santa Maria - RS. R bras ci Saúde 2016:20(2):115-22.

19. Rossi SS, Santos PG, Passos JP. A sindrome de Burnout no enfermeiro: um estudo comparativo entre a Atenção Básica e setores fechados hospitalares. R Pesq Cuid fundam [Internet] 2010 Out-Dez [citado 2018 Abr 25]; 2(4):1232-39. Disponível: http://www.redalyc.org/articulo.oa?id=565750833002. 
20. Leonelli LB, Andreoni S, Martins P, Kozasa EH. Estresse percebido em profissionais da Estratégia Saúde da Família. Rev Bras Epidemiol. 2017 Abr-Jun;20(2):286-98.

21. Carlotto MS. Transtornos mentais comuns em trabalhadores de Unidades Básicas de Saúde : prevalência e fatores associados. Psico Argum. 2016 Abr-Jun;34(85):133-46.

22. Araújo TM, Mattos AIS, Almeida MMG, Santos KOB. Aspectos psicossociais do trabalho e transtornos mentais comuns entre trabalhadores da saúde : contribuições da análise de modelos combinados. Rev bras epidemiol [Internet]. 2016 [citado 2018 Maio 25]:19(3):645-57. Disponivel: http://dx.doi.org/10.1590/19805497201600030014.

23. Albuquerque FJB, Melo CF, Neto JLA. Avaliação da Síndrome de Burnout em Profissionais da Estratégia Saúde da Família da Capital Paraibana. Psicol Reflex Crit. 2012:25(3):542-49.

24. Moreira IJB, Horta JA, Duro LN, Borges DT, Cristofari AB, Chaves J, et al. Perfil sociodemográfico, ocupacional e avaliação das condições de saúde mental dos trabalhadores da Estratégia Saúde da Família em um município do Rio Grande do Sul, RS. Rev Bras Med Fam Comunidade. 2016 Jan-Dez;11(38):1-12. 